\title{
Décès d'un "raveur" pouvant être imputé à un nouveau produit : la 4-Méthylthioamphétamine
}

\author{
Fatal intoxication by a new drug \\ 4-Methylthioamphetamine (4-MTA)
}

\begin{abstract}
Marie-Hélène GHYSEL, Véronique DUPONT, Florence DESCAMPS, Claire LEROUX, Ornella SALVADORE
\end{abstract}

Laboratoire de Police Scientifique, 7, boulevard Vauban - 59800 LILLE

*Auteur à qui adresser la correspondance : Marie-Hélène GHYSEL, Laboratoire de Police Scientifique, 7, boulevard Vauban - 59800 LILLE - Tél : 33 (0)3 20128979 - Fax : 33 (0)32012 8999

\section{RÉSUMÉ}

En avril 1999 le corps sans vie d'un homme de 26 ans, adepte des soirées "raves" est découvert à son domicile. Des cactus coupés sont retrouvés dans l'appartement. Le cadavre est en état de putréfaction avancée. Le médecin légiste conclut que la mort est en rapport avec un adème pulmonaire.

Le cactus coupé, a l'aspect d'un Trichocereus. Après extraction par le chloroforme en milieu alcalin et une analyse par chromatographie en phase gazeuse couplée à un spectromètre de masse ( $C G-S M$ ), de la mescaline est mise en évidence dans le cactus. Le sang prélevé à l'autopsie est analysé par "Espace de tête" CG-DIF, ainsi que, après extraction par Toxi-Tube $B$ A, par CG-SM et par chromatographie liquide couplêe à un détecteur Ultraviolet à Barrette de Diodes (CLHP/BD). Des extractions et dérivations spécifiques de la recherche des opiacés, de la cocaine, du cannabis, de l'amphétamine et des dérivés apparentés à l'ecstasy, de la buprénorphine sont également réalisées sur ce sang. Un nouveau produit, la 4-méthylthioamphétamine, absent des bibliothèques de spectres vendues par les constructeurs est retrouvé dans ce sang. Sa quantification est réalisée par CLHP à $220 \mathrm{~nm}$ après extraction liquide à pH alcalin par un mélange dichlorométhane-hexane-acétate d'éthyle $(50,40,10)$. La colonne est une C8 Symmetry ${ }^{\circledR}$ de chez WATERS, la phase mobile est composée d'un mélange d'acétonitrile et de dihydrogénophosphate de sodium. Les analyses toxicologiques mettent en évidence dans le sang, de la mescaline $(0,1 \mu \mathrm{g} / \mathrm{ml})$ et de la 4 méthylthioamphétamine au taux de $2,11 \mathrm{\mu g} / \mathrm{ml}$. L'alcoolémie est insignifiante $(0,1 \mathrm{~g} / \mathrm{l})$. Les recherches d'autres produits toxiques d'origine organique sont négatives, notamment en ce qui concerne les autres produits stupéfiants ou l'éphédrine. Ce type d'intoxication serait le premier rapporté en France.

\section{MOTS CLES}

4-Méthylthioamphétamine, 4-MTA, cactus, mescaline, décès, analyses toxicologiques.
(Reçu le 24 juillet 2000 ; accepté le 4 septembre 2000)

\section{SUMMARY}

We report here a case of intoxication concer,ling a 26 yearold man who was found dead at home, near sliced cactus. The cactus (Trichocereus) was analyzed and revealed mescaline. Toxicological screening was performed on blood, after extraction using Toxi-Tube®A, and specific extraction for drugs of abuse, followed by identification by gas chromatography coupled with mass spectrometric detection ( $G C$ MS) and high performance liquid chromatography with diode array detection (HPLC-DAD).

Mescaline and 4-methylthioamphetamine, a new designer drug, were found in blood. Quantitation was performed by HPLC at $220 \mathrm{~nm}$ after liquid extraction at $\mathrm{pH} 9$ by dichloromethane-hexane-ethyl acetate $(50,40,10)$. The column used was a C8 Symmetry ${ }^{\circledR}$ WATERS, mobile phase is acetonitrile and sodium dihydrogenophosphate. Blood concentration of mescaline and 4-MTA were respectively 0.1 and $2.11 \mathrm{mg} / \mathrm{l}$. To our knowledge, this case is the first one described in France.

\section{KEY WORDS}

4-methylthioamphetamine, 4-MTA, cactus, mescaline, toxicological analysis, death. 


\section{Introduction}

En avril 1999, un homme de 26 ans, dont la famille était sans nouvelle depuis plusieurs jours est retrouvé mort à son domicile. Les policiers appelés sur les lieux notent la présence de différents détails leur permettant de penser que la victime était un adepte de musique techno et des soirées "raves" et qu'il s'intéressait aux produits "phytopsychoactifs" : des affiches de concerts de musique Hardcore sont accrochées au mur de l'appartement ; ils notent la présence d'un sachet de "herbal energizer", d'une paille courte semblable à celle utilisée par les individus qui sniffent des produits, d'un livre sur les boutons floraux de cannabis, les sinsemilla. Leur attention est également attirée par la présence de cactus de forme allongée, dont l'extrémité est coupée, comme si une tranche de cactus avait été découpée (Figure 1).

A l'autopsie, les médecins légistes notent une putréfaction du corps et un œedème pulmonaire.

La jardinière contenant le cactus coupé et le prélèvement de sang réalisé à l'autopsie sont amenés au laboratoire pour analyse.

\section{Examen du cactus}

L'examen botanique du cactus (forme, emplacement des épines sur des côtes saillantes de la tige charnue...) indique qu'il peut s'agir d'un trichocereus.

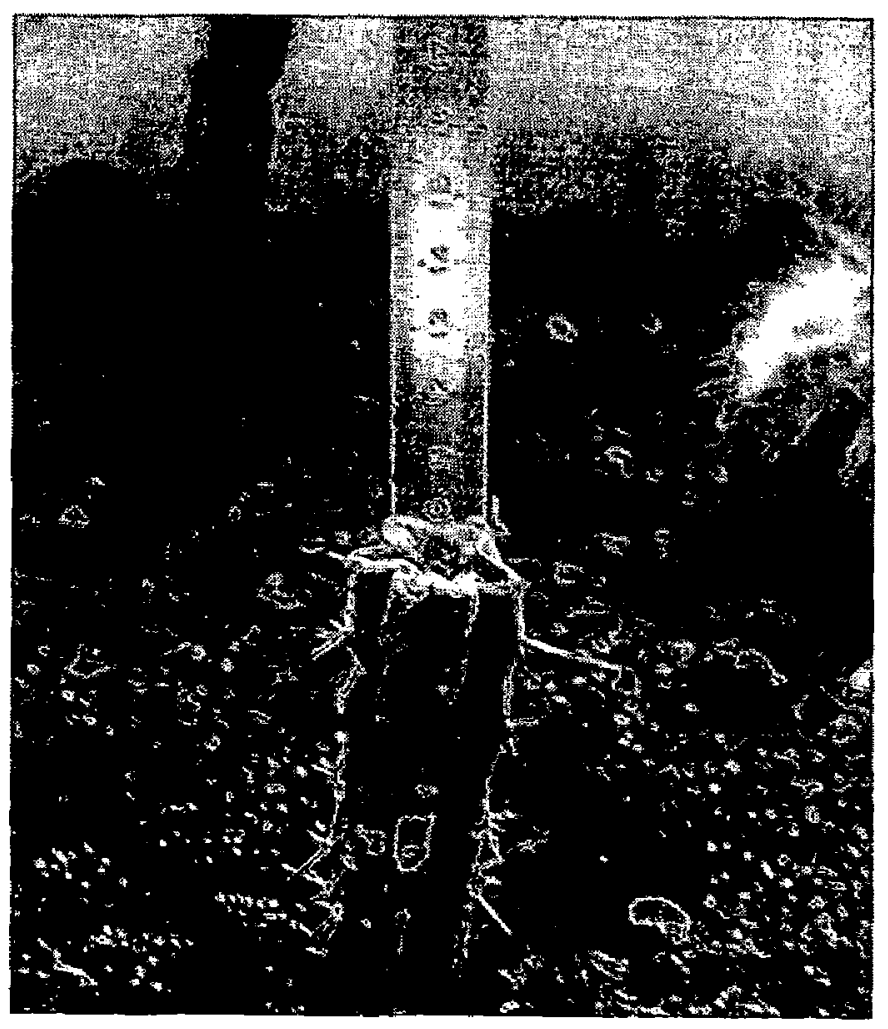

Figure 1 : Photographie du Trichocereus coupé.

\section{Analyses \\ Réactifs}

Le chloroforme, l'isopropanol, le méthanol, l'acétonitrile, le dichlorométhane, l'hexane, l'acétate d'éthyle, le chlorobutane, le toluène sont de qualité HPLC. Les Toxi-Tube $^{\oplus} \mathrm{A}$ viennent de chez ANSYS $^{\circledast}$ (Amilabo, Chassieux, France). Le dihydrogénophosphate de sodium de qualité très pure et la pyridine proviennent de chez MERCK (Damstardt, Allemagne). L'acide chlorhydrique est de qualité analytique.

Les agents dérivants utilisés pour la recherche spécifique des produits stupéfiants sont l'anhydride trifluoroacétique (Fluka, Buchs, Suisse), le tétraméthylammoniumhydroxide pentahydrate (Fluka, Buchs, Suisse), le diméthylsulfoxide (Sigma-Aldrich, Steinheim, Allemagne), l'iodure de méthyle (SigmaAldrich, Steinheim, Allemagne),

le bistriméthylsilyltrifluoroacétamide avec $1 \%$ de triméthylchlorosilane (Fluka, Buchs, Suisse), le triméthylsilyl-imidazole (Sigma-Aldrich, Steinheim, Allemagne), la méthylsilyltrifluoroacétamide (Fluka, Buchs, Suisse), l'anhydride heptafluorobutyrique (Sigma-Aldrich, Steinheim, Allemagne),

Les étalons deutérés viennent de chez Promochem (Molsheim, France). Quant aux produits témoins, la mescaline provenait des Laboratoires Latéma (Suresnes, France), et pour la 4-MTA, quelques milligrammes nous avez été gracieusement donnés par Madame A. Poortman (Forensic Science Laboratory, Rijswijk, Pays-Bas) avant que ce produit ne figure sur la liste des produits stupéfiants.

\section{Instrumentation}

L'alcoolémie est réalisée par chromatographie en phase gazeuse selon la technique de l'espace de tête avec un appareil de marque Sigma 2000 PERKIN ELMER, équipé d'une colonne Porapak Q. La détection se fait par ionisation de flamme.

\section{Le chromatographe en phase gazeuse couplé à un spectromètre de masse (CG-SM)}

L'appareil est un MD800 de marque FISONS (Thermo Quest, Les Ulis, France), la colonne une BPX5 (SGE, Villeneuve Saint Georges, France) de 25 mètres, de $0,22 \mathrm{~mm}$ de diamètre interne et de $0,25 \mu \mathrm{m}$ d'épaisseur de film. L'injection est réalisée en mode splitless. La température de l'injecteur est de $250^{\circ} \mathrm{C}$. La température du four est de $40^{\circ} \mathrm{C}$ pendant une minute, de $40^{\circ} \mathrm{C}$ à $180^{\circ} \mathrm{C}$ à raison de $25^{\circ} \mathrm{C} / \mathrm{min}$ puis de $10^{\circ} \mathrm{C} / \mathrm{min}$ jus- 
qu'à $280^{\circ} \mathrm{C}$ où elle est maintenue pendant 15 minutes. Le débit du gaz vecteur (hélium) est de $1 \mathrm{ml} / \mathrm{min}$. La température de l'interface est de $315^{\circ} \mathrm{C}$. La détection est réalisée par impact électronique à $70 \mathrm{eV}$ avec balayage de 35 à 650 unités de masse atomique. L'acquisition des données est effectuée en mode "spectre complet".

Le chromatographe en phase liquide couplé à un détecteur ultraviolet à barrette de diodes (CLHP/BD)

La pompe est de marque Merck (Nogent sur Marne, France), modèle L6200A, l'injecteur est une vanne manuelle de marque Rhéodyne modèle 7125 avec boucle de $20 \mu \mathrm{l}$. La colonne est une C8 Symmetry ${ }^{\oplus}$ (WATERS, Saint Quentin en Yvelines, France) de $250 \mathrm{~mm} \times 4,6 \mathrm{~mm}$ D.I $(5 \mu \mathrm{m}$ de diamètre des particules). Le détecteur à barrette de diodes est un SM5000 (LDC, Thermo Quest, Les Ulis, France) pouvant enregistrer des spectres de 200 à $360 \mathrm{~nm}$. La phase mobile est un mélange d'acétonitrile, et de dihydrogénophosphate de sodium, selon les conditions décrites (1).

\section{Analyse du cactus}

Un prélèvement de la plante à analyser est mis en macération dans $10 \mathrm{ml}$ de chloroforme en milieu alcalin. L'extrait est évaporé, repris par du méthanol et injecté dans le CG/SM dans les conditions précédemment décrites.

\section{Analyses toxicologiques}

L'alcoolémie est réalisée par chromatographie en phase gazeuse selon la technique de l'espace de tête sur $0,5 \mathrm{ml}$ de sang. Le $\mathrm{N}$-propanol est utilisé comme étalon interne.

Les recherches de toxiques d'origine organique sont réalisées après différents types d'extraction :

$>2 \mathrm{ml}$ de sang sont introduits dans un Toxi-Tube ${ }^{\oplus} \mathrm{A}$ (ANSYS ${ }^{\oplus}$ Amilabo, Chassieux, France). Après agitation pendant 5 minutes et centrifugation pendant 10 minutes, la phase organique est évaporée à sec. Le résidu est repris par $100 \mu \mathrm{l}$ de méthanol, $2 \mu \mathrm{l}$ sont analysés par CG/SM et $20 \mu \mathrm{l}$ par CLHP/BD.

$>1 \mathrm{ml}$ de sang est soumis à une extraction spécifique de la recherche des opiacés et de la cocaïne en présence d'une solution titrée des étalons internes deutérés (morphine-d3, codéine-d3, 6-monoacéthylmorphine$\mathrm{d} 3$, cocaïne-d3, benzoylecgonine-d3, cocaéthylène-d3, ecgonineméthylester-d3, Promochem, Molsheim, France). L'extraction est réalisée par un mélange chloroforme/isopropanol (9/1) après passages successifs en milieu basique ( $\mathrm{pH} \mathrm{8,4)} \mathrm{acide} \mathrm{puis} \mathrm{basique}(\mathrm{pH} \mathrm{8,4).} \mathrm{La}$ phase organique finale est évaporée à sec. Le résidu est dérivé par $50 \mu \mathrm{l}$ de Bistriméthylsilyltrifluoroacétamide $+1 \%$ de Triméthylchlorosilane (BSTFA-TMCS) à $70^{\circ} \mathrm{C}$ pendant $20 \mathrm{~min}$.

$>1 \mathrm{ml}$ du sang est soumis à une extraction spécifique de la recherche des amphétamines en présence d'une solution titrée des étalons internes deutérés ( amphétamine-d5, méthylènedioxyamphétamine- $\mathrm{d} 5$, méthylènedioxyéthamphétamine- $\mathrm{d} 5$, méthamphétamine- $\mathrm{d} 8$, Promochem, Molsheim, France. L'extraction est réalisée au chlorobutane en milieu basique. La phase organique est dérivée par $75 \mu \mathrm{l}$ d'anhydride Trifluoroacétique (TFAA), en présence de $150 \mu \mathrm{l}$ de pyridine, chauffé $30 \mathrm{~min}$ à $50^{\circ} \mathrm{C}$.

$>2 \mathrm{ml}$ de sang sont soumis à une extraction spécifique de la recherche des cannabinoïdes en présence d'une solution titrée des étalons deutérés (delta-9-tétrahydrocannabinol-d3, 11-nor-9-carboxy-delta-9-THC-d3, Promochem, (Molsheim, France). L' extraction est réalisée par un mélange d'hexane/acétate d'éthyle $(9 / 1, v / v)$, en milieu acide. La phase organique est évaporée. Le résidu est dérivé en présence de $200 \mu \mathrm{l}$ de TétraMéthylAmmoniumHydroxide/DiméthylSulfoxide (1/20) (TMAH/DMSO), et $50 \mu$ d'iodure de méthyle, selon les conditions publiées(2).

$>1 \mathrm{ml}$ est prélevé et soumis à une extraction spécifique de la recherche de buprénorphine en présence de buprénorphine-D4 (Promochem, Molsheim, France). L'extraction est réalisée par du toluène, après passage en milieu basique, acide puis basique, la phase organique finale est évaporée à sec. Le résidu est dérivé par un mélange de $50 \mu \mathrm{l}$ de Méth y lSil y $1 \mathrm{Tr}$ ifluoroAcét a mideTriMéthylChloroSilane-TriméthylSilylImidazole. (MSTFA /TMCS/TSIM) à $65^{\circ} \mathrm{C}$ pendant $20 \mathrm{~min}$. Les extraits sont analysés par CG/SM.

\section{Résultats}

De la mescaline est mise en évidence dans le cactus par CLHP-BD (dans les conditions précédemment décrites le temps de rétention est de $6,3 \mathrm{~min}$, le spectre UV est conforme à celui déjà publié(3)), ainsi que par CG-SM avec les fragments $\mathrm{m} / \mathrm{z}$ caractéristiques : 182,167 , $181,211$.

L'analyse du sang a donné les résultats suivants : l'alcoolémie est insignifiante : $0,1 \mathrm{~g} / \mathrm{l}$. Les recherches spécifiques des opiacés, de la cocaïne, du delta-9 tétrahydrocannabinol, de la buprénorphine et de leurs métabo- 
lites sont négatives. Les recherches de l'amphétamine, la méthamphétamine, de la méthylènedioxyméthamphétamine (MDMA), de la méthylènedioxéthamphétamine (MDEA), de la méthylènedioxyamphétamine (MDA), de la méthylbenzodioxazolylbutanamine (MBDB), de la 4-bromo-2,5diméthoxyphenéthylamine (2C-B), de la 4-bromo-2,5diméthoxyamphétamine (DOB), de 1'éphédrine et pseudoéphédrine sont négatives.

De la mescaline est mise en évidence dans le sang par CG-SM et par CLHP avec des temps de rétention dans nos conditions opératoires respectivement de 9,9 et 6,6 min (A titre indicatif, dans nos conditions opératoires les temps de rétention de la caféine sont de 12,6 et $6,9 \mathrm{~min}$ )

Après injection dans le CG/SM de l'extrait sanguin réalisé à partir du Toxi-Tube ${ }^{\circledast} \mathrm{A}$, un pic présentant un spectre de masse correspondant à un nouveau produit récemment signalé(4), la 4-méthylthioamphétamine (4MTA) est également mis en évidence, par son spectre de masse obtenu par impact électronique( Figure 2) et son spectre UV (Figure 3). Les temps de rétention après CG-SM et par CLHP dans nos conditions opératoires sont respectivement de 9,1 et $10,6 \mathrm{~min}$ alors que celui de la caféine est de 12,6 et $6,9 \mathrm{~min}$.

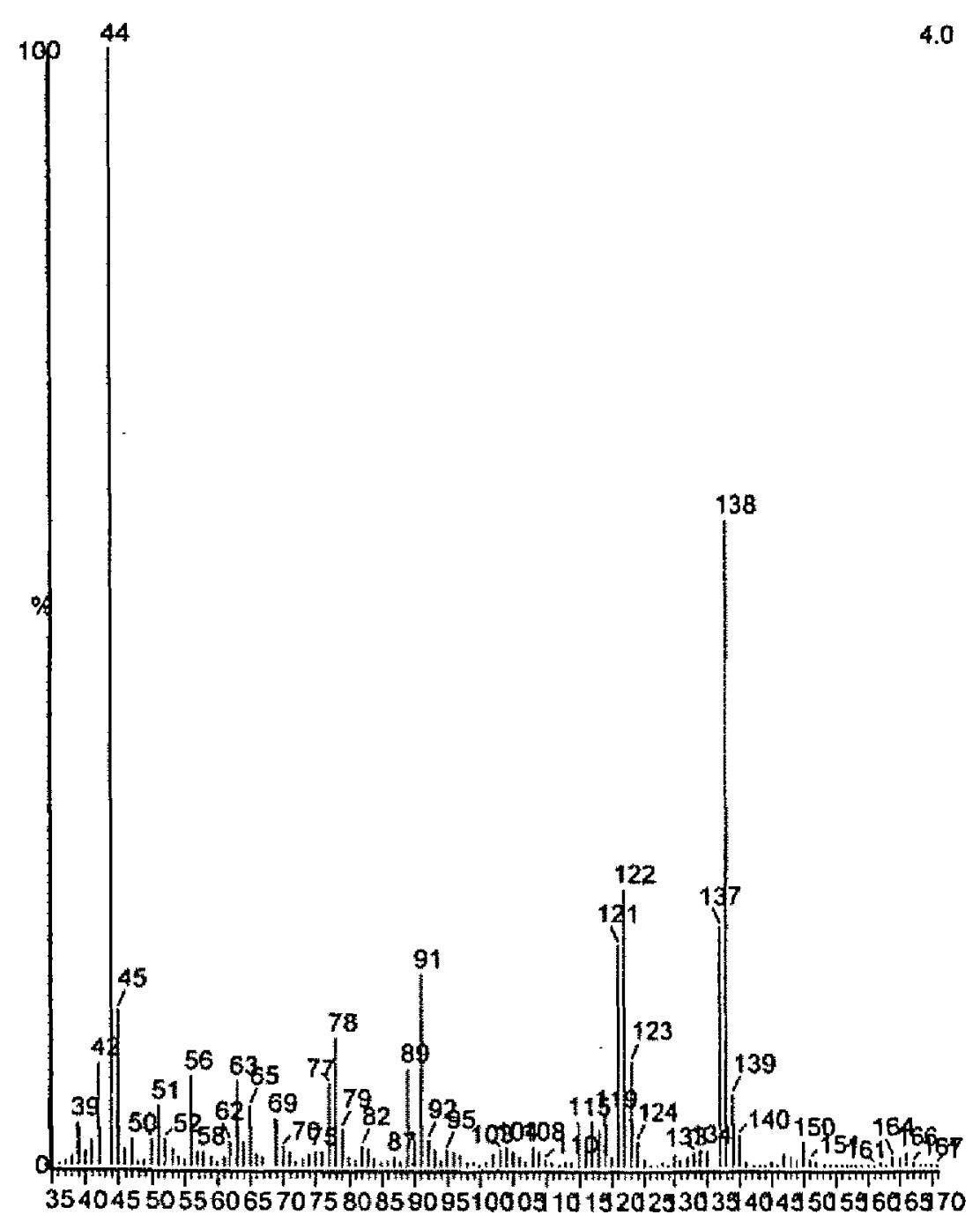

Figure 2 : Spectre de masse de la 4-MTA.

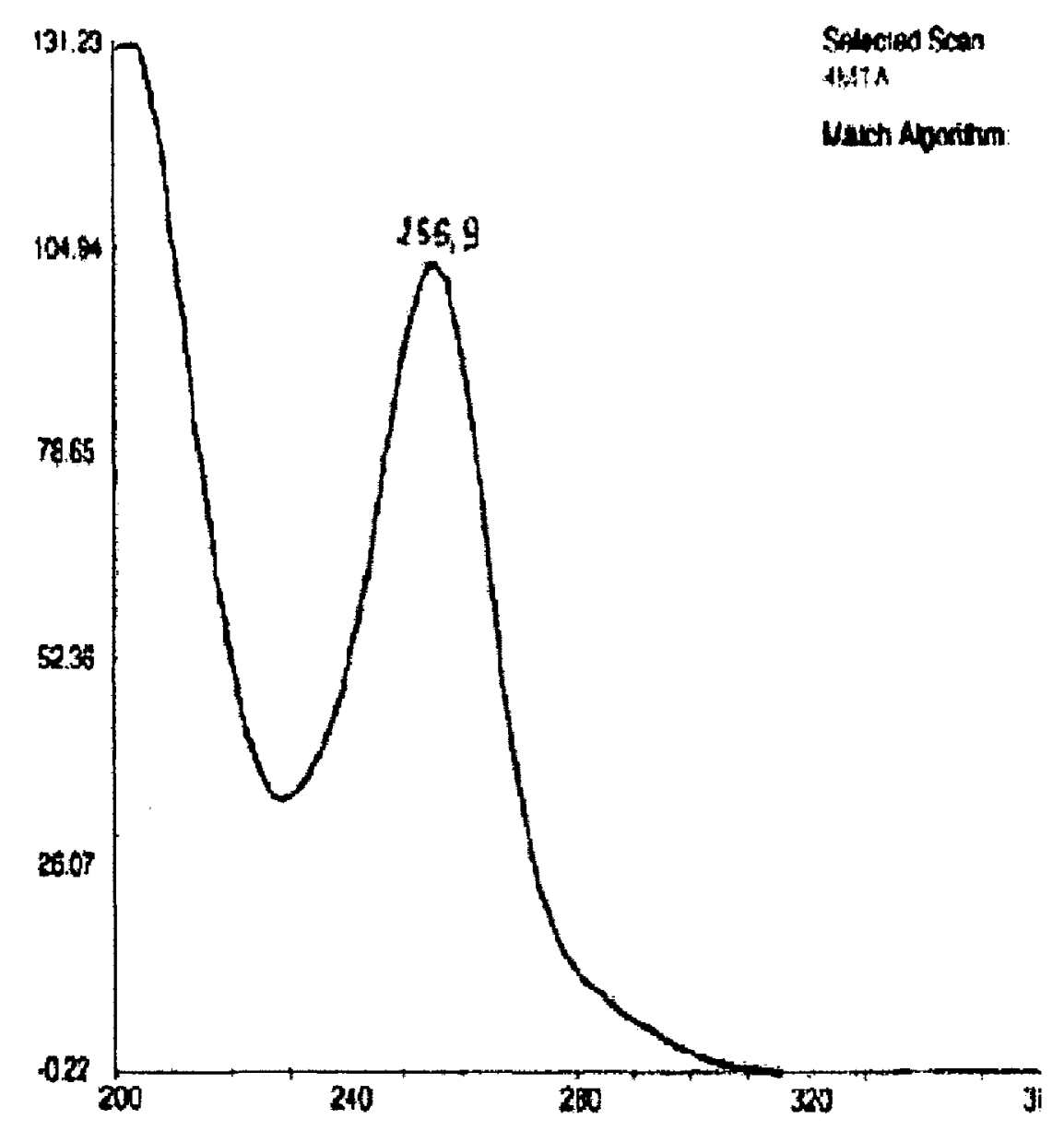

Figure 3 : Spectre Ultra-violet de la 4-MTA.

Le dosage de la 4-MTA et de la mescaline a été réalisé dans le sang. La droite d'étalonnage obtenue après ajout de quantité croissante de 4-MTA à la concentration de 1,$2 ; 1,9 ; 3,4 ; 4,2 ; 6,4$; et $10 \mathrm{mg} / 1$ et de mescaline à la concentration de 60,200 et $250 \mu \mathrm{g} / \mathrm{l}$ dans du sang vierge et extraction par Toxi-Tube ${ }^{\Theta} \mathrm{A}$ ne donnant pas de résultats satisfaisants, les extractions ont été réalisées en milieu alcalin $(180 \mu \mathrm{l}$ de $\mathrm{NaOH} 0,1 \mathrm{M}$ pour 1 $\mathrm{ml}$ de sang) par $2 \mathrm{ml}$ d'un mélange dichlorométhanehexane-acétate d'éthyle $(50,40,10)$.

Les extraits sont analysés par CLHP-BD dans les conditions précédemment décrites (1). La quantification est réalisée à $220 \mathrm{~nm}$.

De la mescaline et de la 4-MTA aux taux respectifs de 0,1 et $2,11 \mathrm{mg} / \mathrm{l}$ sont retrouvés dans le sang. Aucune autre substance médicamenteuse ou toxique n'est retrouvée avec les techniques analytiques utilisées.

\section{Discussion}

La mescaline est un alcaloïde présent dans des cactés. Le plus connu étant le Peyotl, cependant elle est aussi présente dans le Trichocereus (5) où elle se trouverait dans certaines variétés à la concentration de $2 \%$ du poids sec ou $0,12 \%$ de la plante fraîche (6).

A notre connaissance, il n'y a pas eu de décès dî seulement à la mescaline si ce n'est celui d'un homme 
ayant pris de la mescaline et qui serait mort des suites d'une chute de 600 pieds. Les taux sanguins, hépatiques et urinaires étaient respectivement de $9,7 \mathrm{mg} / 1$; $71 \mathrm{mg} / \mathrm{kg}$; et $1163 \mathrm{mg} / 1$ (7).

Le taux obtenu dans le cas présenté était faible, à la limite de quantification par cette méthode dans le sang. Le dosage de la mescaline dans le sang avait été réalisé par CLHP pour des raisons de disponibilité des appareils, cependant, il apparait, d'après les résultats publiés par Kintz et coll. sur des urines supplémentées en mescaline (3), que la limite de détection est plus basse par CG-SM après dérivation au HFBA.

La 4-MTA est un produit synthétisé par Nichols en 1992 dans le cadre de recherche de produits libérateurs de sérotonine non neurotoxiques (8). Sa formule chimique est présentée figure 4 . Elle a un poids moléculaire de 181,3 et son numéro de CAS est 14116-06-04. (La 4-MTA n'a pas été mise en évidence dans le cactus analysé.)

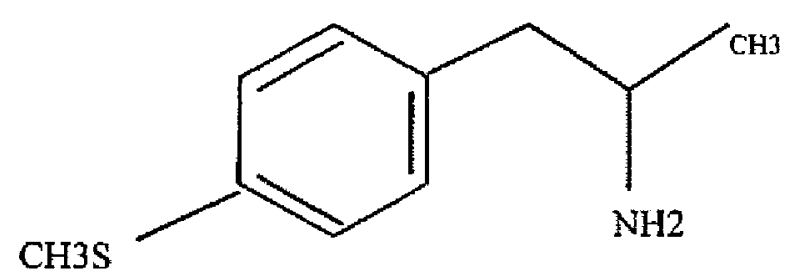

Figure 4 : Formule de la 4-MTA.

Elle est également appelée p-MTA ou "flatliner"(électrocardiogramme plat). Elle est vendue sous forme de comprimés soit sans logo, soit portant l'inseription "S5" $(9,10)$, "MK" ou encore un comprimé de couleur rose portant un logo représentant une étoile à 5 branches. Des saisies importantes de comprimés avaient été réalisées en 1998 en Belgique, au Royaume-Uni et au PaysBas. Il n'y a pas eu de saisies majeures signalées en France, mais plutôt des comprimés isolés (11).

Peu d'études pharmacologiques ont été réalisées pour l'instant sur la 4-MTA. D'après celles qui existent, elle augmente la libération de sérotonine dans le cerveau, inhibe la recapture de la sérotonine, ainsi que la monoamine oxydase (12). Ses effets seraient plus tardifs que ceux obtenus avec l'ecstasy. Elle peut provoquer de ce fait davantage d'intoxications chez ceux qui pensent consommer un produit peu concentré et qui ainsi augmentent la dose pour être plus rapidement sous influence. Au point de vue pharmacocinétique, les études récemment présentées par Elliott font état d'une demi-vie évaluée à 7 heures et d'une métabolisation possible en dérivé sulfoxy(13).

Du point de vue analytique, dans le cas présenté, la 4MTA a été mise en évidence dans le sang après extraction alcaline sans dérivation. Des essais ont été réalisés après dérivation au HFBA et au TFAA. Les spectres de masse de cette 4-MTA dérivée par ces deux produits sont respectivement joints figures $n^{\circ} 5$ et $n^{\circ} 6$.

Dans la bibliographie, on retrouve qu'elle donne un résultat positif avec le test d'immunoanalyse EMIT ${ }^{{ }_{-}}$ amphétamine à $1 \mu \mathrm{g} / \mathrm{ml}$, et ceci jusqu'à 23 heures dans les urines après administration à un chien (14). Elle a également positivé les immuno-essais urinaires après analyse par polarisation de fluorescence (FPIA) amphetamine/methamphetamine II de l'analyseur $\mathrm{ADX}^{\circledast}$ Abbott et l' EMIT $^{\circledast}$ d.a.u. monoclonal amphetamine/methamphetamine du Syva ETS ${ }^{\circledR}$ analyser. (15)

Quant à la mise en évidence de la 4-MTA dans les saisies de comprimés, par réaction colorée, chromatographie sur couche mince, spectroscopie infra-rouge, CG$\mathrm{SM}$, Résonance magnétique nucléaire ( $\mathrm{RMN})$, cela a fait l'objet de plusieurs publications $(4,16,17)$

Les concentrations sanguines retrouvées dans d'autres

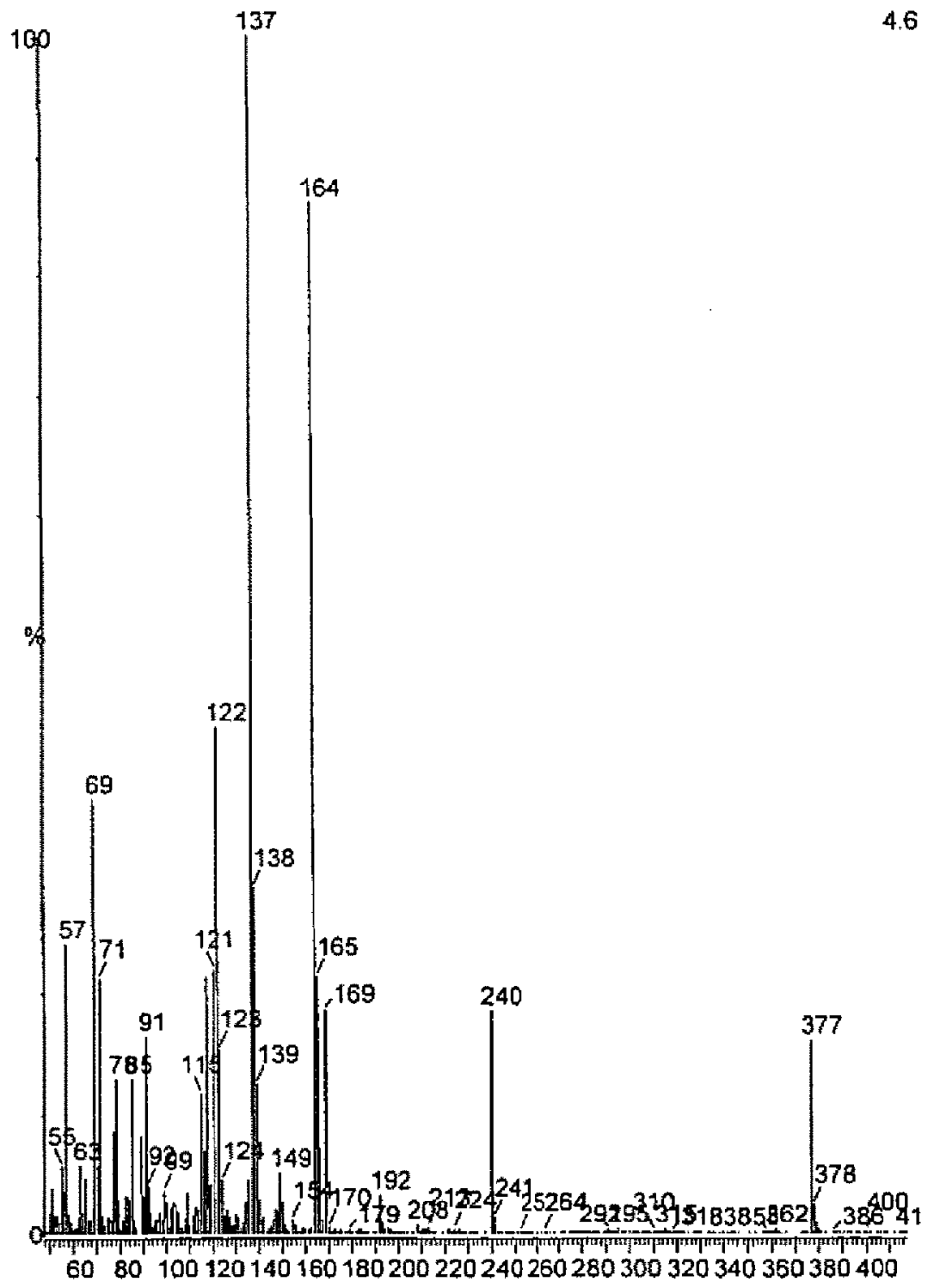

Figure 5 : Spectre de masse de la 4-MTA-HFBA. 


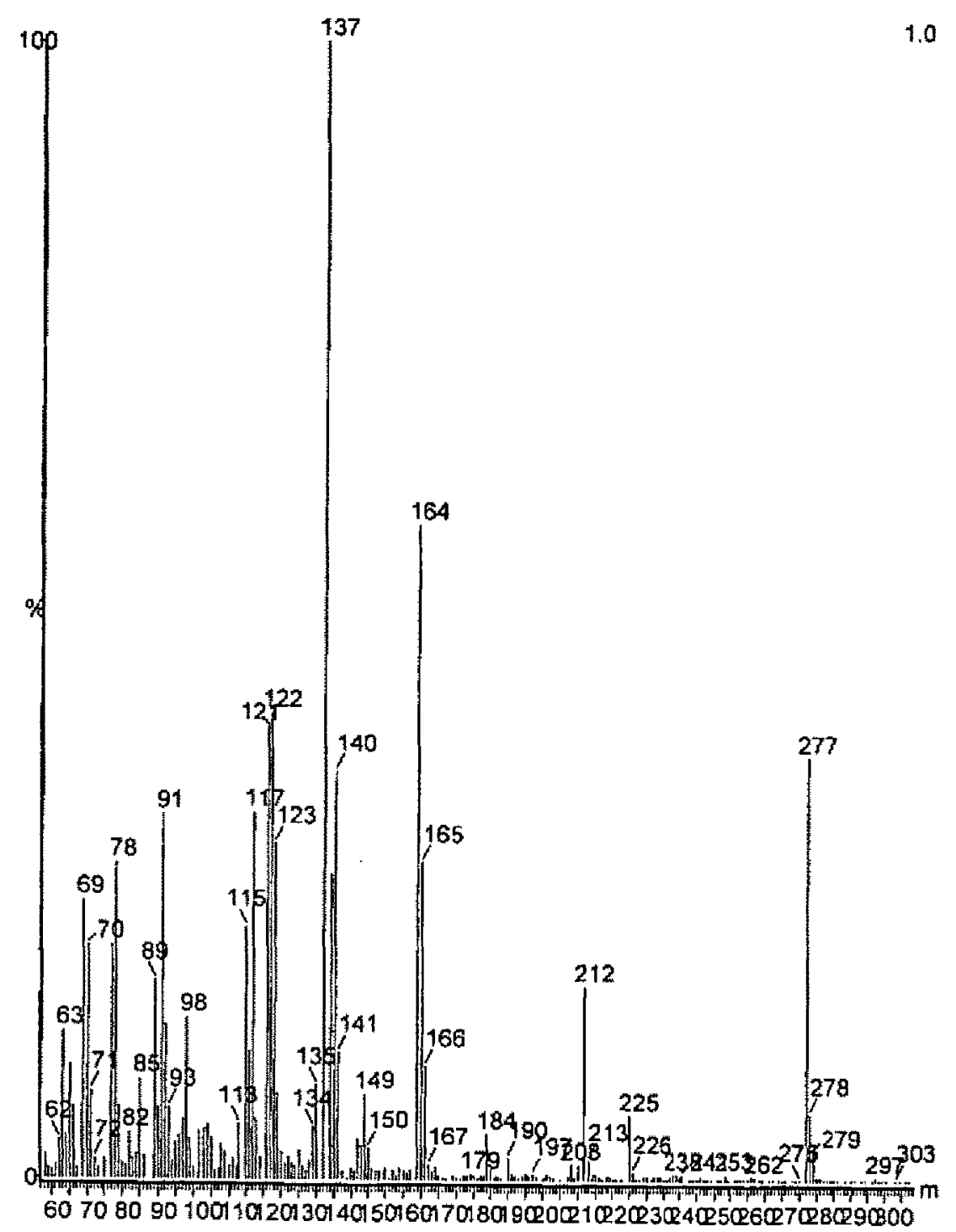

Figure 6 : Spectre de masse de la 4-MTA-TFA.

cas d'intoxication par la 4-MTA, rapportées par Elliott $(18,19)$ sont reprises dans le tableau I.

Le rapport entre les taux sanguins et la toxicité a été évaluée par S. P. Elliott (18); ainsi, de 200 à $600 \mathrm{ng} / \mathrm{ml}$ la toxicité serait modérée ; au dessus de $600 \mathrm{ng} / \mathrm{ml} \mathrm{la}$ toxicité est sévère, quant aux concentrations supérieures à $1500 \mathrm{ng} / \mathrm{ml}$ elles correspondraient à une intoxication potentiellement létale.

Tableau I : Concentrations sanguines de 4-MTA obtenues dans différentes analyses toxicologiques d'après S.P.Elliott $(18,19)$.

\begin{tabular}{|c|c|c|c|}
\hline Date & Produits retrouyés & $\begin{array}{l}\text { Concentration sanguine } \\
\text { de 4-MTA (mg/) }\end{array}$ & Etat du sujet \\
\hline $07 / 98$ & 4-MTA et MDMA & 0,19 & Vivant \\
\hline $07 / 98$ & 4-MTA & $\begin{array}{c}\text { 4,20 pendant la } \\
\text { réanimation } \\
4,60 \text { sang fémoral post } \\
\text { mortem }\end{array}$ & Décédé \\
\hline $08 / 98$ & $\begin{array}{c}\text { 4-MTA, } \\
\text { amphétamine } \\
\text { pseudo-éphédrine }\end{array}$ & 0,13 & Vivant \\
\hline $09 / 98$ & $\begin{array}{l}\text { 4-MTA, } \\
\text { amphétamine } \\
\text { MDMA }\end{array}$ & 0,76 & Vivant \\
\hline
\end{tabular}

\section{Conclusions}

Les adeptes des produits psychoactifs sont toujours à la recherche de nouveaux produits. Ce phénomène est très préoccupant pour les acteurs de santé publique et à chaque fois un challenge pour les toxicologues.

Ce décès serait le premier décrit en France, d'autres s'étant déjà produits au Pays-Bas et au Royaume-Uni en 1997 et 1998. Le centre européen de prévention des médicaments et des drogues a fait réaliser une étude sur les risques de ce produit (12). A la suite de quoi, la 4MTA a été classée dans la liste des produits stupéfiants dans de nombreux pays européens dont la France le 24 novembre 1999.

\section{Références}

1. Gaillard Y., Pépin G. Use of high-performance liquid chromatography with photodiode array UV detection for the creation of a 600-compound library. Application to forensic toxicology. J. Chromatogr. A. $1997 ; 763$ : 149163.

2. Kintz P., Cirimele V., Pépin G., Marquet P., Deveaux M., Mura P. Identification et dosage des cannabinoïdes dans le sang total. Toxicorama $1996 ; 8: 29-33$.

3. Kintz P., Cirimele V., Tracqui A., Ludes B. Peyotl : le cactus divin. Toxicorama $1998 ; 10: 91-94$.

4. Poortman A.J. The identification of 4-methylthioamphetamine. Microgram. 1998 ; 31 : 174-180.

5. Bruneton J. Plantes toxiques. Végétaux dangereux pour l'homme et les animaux. Lavoisier Tec \& doc. Paris, $1996: 207-209$.

6. Schultes R.E., Hofman A. The botany and chemistry of hallucinogens. Springfield : Charles $\mathrm{C}$ Thomas Publisher, $1980 ; 225-227$.

7. Reynolds P.C., Jindrich E.J.. A mescaline associated fatality. J. Anal. Toxicol. $1985 ; 9: 183-184$.

8. Huang X., Marona-Lewicka D., Nichols D. E.. p-Methylthioamphetamine is a potent new neurotoxic serotonin releasing agent. Eur. J. Pharmacol. $1992 ; 229$ : 31-38.

9. De Boer D., Egberts T., Maes R.A. Para-methylthioamphetamine, a new amphetamine designer drug of abuse. Pharmacy world \& Science $1999 ; 21(1): 47-48$.

10. Bosman I.J., De Boer D., Siderius E.B., Dos Reys L.J.,Maes R.A.A. Mass spectrometric identification of some sulfur containing phenalkylamine designer drugs. Problems of Forensic Sciences. 2000 ; XLII : 215-220.

11. Arditi J., Bourdon J.H., Beauverie P., Gatignol C., Sarradet A. Les substances consommées dans 'l'espace festif techno" Résultats préliminaires d'un laboratoire participant au système d'identification nationale des toxiques et substances. Communication affichée au congrès annuel de la Société Française de Toxicologie Analytique (SFTA) les 7, 8 et 9 juin 2000 à Limoges, p 222 des résumés 
12. Anonyme. Report on the risk assessment of 4-MTA in the framework of the joint action on new synthetic drugs. EMCDDA, Office for official Publications of the european communities. Luxembourg, 1999.

13. Elliott S.P. Analysis of 4-Methylthioamphetamine (4MTA) in clinical specimens. Proceeding of the 38 th meeting of The International Association of Forensic Toxicologist, Helsinki, Finland, 2000 in press.

14. Kavanagh P.V., Corrigan D., Maguire R.T., Meegan M.T., Keating J.J., Clancy J., Burdett J. Excretion profile of 4-methylthioamphetamine in dogs. Pharmacy and pharmacology communications. 1999, 5/11:653-655.

15. Bosman I.J., De Boer D., Maes R.A.A. Screening for the presence of para-méthylthioamphetamine in urine by some commercial immunoassays and confirmation by GC/MS. Problems of Forensic Sciences. 2000 ; XLIII : 51-56.
16. Groombridge C.J. The identification of 4Methylthioamphetamine in a drug seizure. Microgram $1998 ; 31: 150-155$.

17. Poortman A.J., Lock E. Analytical profile of 4-methylthioamphetamine (4-MTA), a new street drug. Forensic Sci. Int. $1999 ; 100: 221-233$.

18. Elliott S.P. An initial review of analytical findings in cases involving 4-methylthioamphetamine (4-MTA). Bulletin du TIAFT $1999 ; 29$ (2) : 7-9.

19. Elliott S.P. Fatal poisoning with a new phenylethylamine : 4-methylthioamphetamine (4-MTA) J. Anal. Toxicol. $2000 ; 24$ (2) : 85-89. 